\title{
Upregulated unique long 16 binding protein 1 detected in preeclamptic placenta affects human extravillous trophoblast cell line (HTR-8/SVneo) invasion by modulating the function of uterine natural killer cells
}

\author{
JING LIU ${ }^{1}$, GUANG SONG ${ }^{2}$, XUEWEN LIN $^{3}$, XINING PANG $^{3}$ and TAO MENG ${ }^{1}$ \\ Departments of ${ }^{1}$ Obstetrics, ${ }^{2}$ Ultrasound and ${ }^{3}$ Stem Cells and Regenerative Medicine, \\ The First Affiliated Hospital of China Medical University, Shenyang, Liaoning 110001, P.R. China
}

Received December 10, 2015; Accepted December 23, 2016

DOI: $10.3892 / \mathrm{etm} .2017 .4143$

\begin{abstract}
Well-controlled trophoblast invasion at the maternal-fetal interface is crucial for normal placentation and successful pregnancy, otherwise pathological conditions of pregnancy occur, such as preeclampsia. In previous studies, it has been demonstrated that unique long 16 binding protein (ULBP)1, a ligand for the natural-killer group (NKG)2D receptor on uterine natural killer (uNK) cells, is upregulated in the placenta in patients with preeclampsia. As they are present on the majority of the decidua, uNK have an important role in pregnancy. The aim of the present study was to determine the role of ULBP1 in trophoblast cell invasion, which is closely associated with the occurrence of preeclampsia. In the present study, ULBP1 expression levels in placentas collected after cesarean section from women with preeclampsia and normal pregnant women were determined by immunohistochemistry, reverse transcription-quantitative polymerase chain reaction and western blotting. The effects of ULBP1 on extravillous trophoblast cell line (HTR-8/SVneo) invasion mediated via uNK cells and the underlying mechanisms were investigated. mRNA and protein expression levels of ULBP1 were significantly upregulated $(\mathrm{P}<0.05)$ in preeclamptic placentas compared with normal controls. ULBP1 inhibited HTR-8/SVneo cells via the regulation of biological functions of uNK cells, including the downregulation of NKG2D expression on uNK cells and the stimulation of production of cytokines and chemokines that affect extravillous cytotrophoblast invasion by uNK cells. ULBP1 may have an important role in the pathophysiology of preeclampsia through
\end{abstract}

Correspondence to: Professor Tao Meng, Department of Obstetrics, The First Affiliated Hospital of China Medical University, 155 Nanjing North Street, Shenyang, Liaoning 110001, P.R. China

E-mail: mengtao201011@163.com

Key words: preeclampsia, unique long 16 binding protein 1, uterine natural killer cells, extravillous trophoblast invasion the modification of biological functions of uNK cells, which may affect trophoblast invasion.

\section{Introduction}

Preeclampsia is a heterogeneous pregnancy disorder that may develop in late pregnancy, and is characterized by hypertension and proteinuria which may occur after 20 weeks of gestation. It affects $2-8 \%$ of pregnancies worldwide $(1,2)$. Preeclampsia has preclinical (symptomless) and clinical (symptomatic) stages. Until recently, only the symptomatic final stage preeclampsia could be detected by clinical screening. Although extensive research on the pathophysiology of the disease has been conducted, the etiology of preeclampsia remains poorly understood. A two-stage disorder theory is accepted for preeclampsia: Stage 1, poor placentation at the early stage of pregnancy; and stage 2, placental oxidative stress at the late stage of pregnancy. Stage 1 is preclinical and characterized by faulty trophoblast invasion and spiral artery (SA) remodeling, resulting in failure to remodel the SAs supplying the uteroplacental circulation, and placental hypoxia (3).

Trophoblast differentiation, invasion and SA remodeling are regulated by several trophoblast- and/or decidua-derived factors, including cytokines, growth factors, hormones and oxygen, in the first trimester (4-6). Recent studies have reported that impaired invasion of trophoblasts, and the subsequent incomplete SA remodeling, may result in preeclampsia $(6,7)$. Consequently, elucidating the trophoblast invasion mechanisms is crucial in understanding the pathophysiological mechanisms of preeclampsia. The decidual leukocyte population, in particular uterine natural killer (uNK) cells, is proposed to have a major role in the regulation of trophoblast invasion $(8,9)$. Reduced numbers of uNK cells are observed in patients with preeclampsia and intrauterine growth restriction (10), which are conditions associated with poor SA remodeling and reduced trophoblast invasion in the decidua (11).

Unique long 16 binding proteins (ULBPs) which are also termed as retinoic acid early transcripts (RAET), encoded by RAET1 genes, are a family of ligands for natural-killer group 2D (NKG2D) receptors in humans that are frequently 
expressed by tumor cells and mediate biological functions of NK cells (12). The majority of studies of NKG2D ligands have traditionally demonstrated their expression only on infected or transformed cells $(13,14)$; whereas other studies have demonstrated that several normal cells and tissues also express NKG2D ligands $(15,16)$. Constitutive intracellular presence of ULBP1-4 has been recently described in normal human bronchial epithelium (17). A study by Hedlund et al (18) demonstrated that ULBP1-5 are constitutively transcribed and expressed as proteins in human early placenta (8-16 weeks), and have localized expression on the membrane of exosomes of the multivesicular late endosomes in the syncytiotrophoblast (STB). A previous study using DNA microarray analysis and validation by reverse transcription-quantitative polymerase chain reaction (RT-qPCR), demonstrated that ULBP1 was upregulated in preeclamptic placentas (19).

Considering that inadequate invasion of trophoblasts in the first trimester may lead to preeclampsia and the role of uNK cells in the regulation of trophoblast invasion, it was hypothesized that ULBP1 may inhibit the invasion of extravillous trophoblasts (EVTs) by altering cytokines secreted by uNK cells via binding to NKG2D. Although the differential expression of ULBP1 in preeclampsia in the first trimester is difficult to determine, the differential expression of genes or proteins detected in full-term placenta may provide an indication to investigate the mechanism. The present study was performed to determine the expression levels of ULBP1 in placentas collected following cesarean section from women with preeclampsia and normal pregnant women. The functions of ULBP1 in trophoblast invasion were also investigated.

\section{Materials and methods}

Ethics statement. Ethical approval was granted by the Ethics Committee of The First Affiliated Hospital of China Medical University (Shenyang, China) and methods were carried out in accordance with the committee guidelines. Informed consent was obtained from all participating patients.

Tissue collection. The present study included 30 pregnant women with preeclampsia and 30 normal pregnant women. Human placental tissues were collected at the time of cesarean section from the Department of Obstetrics between September 2014 and August 2015, The First Affiliated Hospital of China Medical University (Shenyang, China). The clinical characteristics of the patients included in the present study are summarized in Table I. Preeclampsia was diagnosed according to the reported criteria (20). Patients enrolled in the preeclampsia group had no history of pre-existing or chronic hypertension, although they exhibited $\geq 140 \mathrm{mmHg}$ systolic or $\geq 90 \mathrm{mmHg}$ diastolic pressure on two occasions at least $4 \mathrm{~h}$ apart after 20 weeks of gestation and $\geq 300 \mathrm{mg}$ per 24 -h urine collection after 20 weeks of gestation. Chorionic tissues were obtained from four different parts of the placenta, from which the amniotic membrane and maternal decidual tissues were removed. Tissues were frozen and stored at $-80^{\circ} \mathrm{C}$ until use. Decidual samples were obtained from women undergoing elective surgical termination of pregnancy at 12-14 weeks of gestation (as determined by ultrasound measurement of crown rump length or biparietal diameter). Following collection, decidual tissue was immediately suspended in sterile saline, transported to the laboratory and washed two to three times in sterile phosphate-buffered saline (PBS) to remove excess blood.

Immunocytochemistry. Formalin-fixed and paraffin-embedded tissue sections of $4-\mu \mathrm{m}$ thickness were prepared. Immunostaining was performed using a streptavidin-peroxidase method. Sections were incubated with a ULBP1 primary antibody (1:100; sc-33456; Santa Cruz Biotechnology Inc., Dallas, TX, USA) at $4^{\circ} \mathrm{C}$ overnight, followed by a biotinylated goat serum anti-rabbit immunoglobulin (Ig)G secondary antibody (1:100; 0017; Ultrasensitive; Fuzhou Maixin Biotech Co., Fuzhou, China) at room temperature for $10 \mathrm{~min}$. Subsequent to washing with PBS three times, the sections were incubated with horseradish peroxidase-conjugated streptavidin-biotin (1:1:1; 0017; Ultrasensitive; Fuzhou Maixin Biotech Co.) at room temperature for $10 \mathrm{~min}$, developed using 3,3-diaminobenzidine tetrahydrochloride, lightly counterstained with hematoxylin, dehydrated in alcohol and mounted. Sections were observed under an Olympus BX51 microscope (Olympus Corp., Tokyo, Japan). As a negative control, isotype-specific IgG was used instead of primary antibody to exclude nonspecific binding of the secondary antibody.

$R T-q P C R$. Total RNA was extracted from human placental tissues using TRIzol reagent (Ambion; Thermo Fisher Scientific, Inc., Waltham, MA, USA) and were treated with DNase I (Promega Corp., Madison, WI, USA). cDNA was generated from $1 \mu \mathrm{g}$ total RNA using a PrimeScript Reagent kit (Takara Biotechnology Co., Ltd., Dalian, China), according to the manufacturers' instructions. The PCR reaction $(20 \mu \mathrm{l}$ final volume) contained $10 \mu \mathrm{l}$ SYBR Premix Ex Taq, $2 \mu 1$ cDNA, $0.8 \mu \mathrm{l}$ of each primer and $6.4 \mu \mathrm{l}$ double distilled $\mathrm{H}_{2} \mathrm{O}$. qPCR analysis for ULBP1 and GAPDH was performed as follows: $95^{\circ} \mathrm{C}$ for $30 \mathrm{sec}$; 45 cycles of $95^{\circ} \mathrm{C}$ for $5 \mathrm{sec}$; and $60^{\circ} \mathrm{C}$ for $20 \mathrm{sec}$ using a Roche Lightcycler 480 (Roche Diagnostics $\mathrm{GmbH}$, Mannheim, Germany). Gene-specific amplifications were confirmed with melting curve analysis following RT-qPCR. Relative gene expression levels were determined using the threshold cycle method ( $2^{-\Delta \Delta \mathrm{Cq}}$ method) (21) with reference to the endogenous GAPDH control. The following primers were used: ULBP1 forward, 5'-CAGCAGACGATG AGGACATT-3' and reverse, 5'-GACAGAAAGTGGCAG AAGGTG-3'; and GAPDH forward, 5'-CATGAGAAGTAT GACAACAGCCT-3' and reverse, 5'-AGTCCTTCCACGATA CCAAAGT-3'.

Western blotting. Human placental tissues were lysed in a radioimmunoprecipitation assay lysis buffer (Nanjing KeyGen Biotech Co., Ltd., Nanjing, China), and protein concentrations were determined using an Enhanced BCA Protein Assay kit (Beyotime Institute of Biotechnology, Beijing, China). A total of $20 \mu \mathrm{g}$ of proteins were separated by $10 \%$ SDS-PAGE and transferred onto a polyvinylidene difluoride membrane (Merck Millipore, Darmstadt, Germany). Membranes were blocked for $1 \mathrm{~h}$ at room temperature with TBST $(50 \mathrm{mM}$ Tris-HCl, $150 \mathrm{mM} \mathrm{NaCl}, 0.05 \%$ Tween-20, pH 7.0) containing $5 \%$ nonfat dry milk and incubated with primary antibodies against ULBP1 (1:200; sc-33456; Santa Cruz Biotechnology) 
Table I. Clinical characteristics of pregnant women enrolled on the present study.

\begin{tabular}{lccc}
\hline Clinical characteristic & Normal pregnancy $(\mathrm{n}=30)$ & Preeclampsia $(\mathrm{n}=30)$ & P-value \\
\hline Maternal age (years) & $29.50 \pm 3.21$ & $29.8 \pm 3.05$ & 0.71 \\
Body mass index $\left(\mathrm{kg} / \mathrm{m}^{2}\right)$ & $24.82 \pm 1.89$ & $25.67 \pm 1.45$ & 0.06 \\
Systolic blood pressure $(\mathrm{mmHg})$ & $119.67 \pm 9.24$ & $155.05 \pm 10.53$ & $<0.05^{\mathrm{a}}$ \\
Diastolic blood pressure $(\mathrm{mmHg})$ & $68.5 \pm 6.33$ & $105.13 \pm 9.18$ & $<0.05^{\mathrm{a}}$ \\
24 h urine protein $(\mathrm{g})$ & $0.047 \pm 0.025$ & $2.54 \pm 1.40$ & $<0.05^{\mathrm{a}}$ \\
Gestational age at delivery (weeks) & $38.84 \pm 0.54$ & $37.26 \pm 1.91$ & $<0.05^{\mathrm{a}}$ \\
Fetal birth weight $(\mathrm{g})$ & $3433.33 \pm 320.78$ & $2580.00 \pm 600.87$ & $<0.05^{\mathrm{a}}$
\end{tabular}

Data are presented as the mean \pm standard error of the mean. ${ }^{\mathrm{P}}<0.05$, significant difference between normal pregnancy and preeclampsia.

and $\beta$-actin $(1: 8,000 ; 66009-1$; Proteintech Group, Inc., Chicago, IL, USA) overnight at $4^{\circ} \mathrm{C}$. The membranes were washed with TBST three times for $10 \mathrm{~min}$, followed by incubation with horseradish-peroxidase-labeled secondary antibodies (1:12,000; SB-0071; Dingguo, Beijing, China) for $1 \mathrm{~h}$ at room temperature. Protein expression was visualized using an enhanced chemiluminescence system (Tanon5200; Tanon Science and Technology Co., Ltd., Shanghai, China). Bands were analyzed using densitometry with ImageJ software (version 1.46r; National Institutes of Health, Bethesda, MA, USA).

uNK cell isolation. Total decidual cell isolates and purified $\mathrm{CD}^{2} 6^{+} \mathrm{CD}^{-} \mathrm{uNK}$ cell isolates were prepared by enzymatic disaggregation and immunomagnetic selection (MACS) as previously described $(22,23)$. The tissue was trimmed into 1-mm pieces and enzymatically digested for $20 \mathrm{~min}$, using vigorous shaking, with type I DNase and IV collagenase in RPMI 1640 medium (Hyclone; GE Healthcare Life Sciences, Logan, UT, USA). Supernatants were collected and incubated overnight in a tissue culture dish in a standard $37^{\circ} \mathrm{C}$ environment with $5 \% \mathrm{CO}_{2}$. Nonadherent cells were collected and loaded onto Ficoll-Paque density gradient media (GE Healthcare Bio-Sciences, Pittsburgh, PA, USA) to purify the lymphocyte population. Cell suspensions were subjected to MACS (MidiMACS; Miltenyi Biotec $\mathrm{GmbH}$, Bergisch Gladbach, Germany) with an NK Cell Isolation kit (Miltenyi Biotec $\mathrm{GmbH}$ ) to obtain uNK cell suspensions. uNK cells were plated on a 24 -well plate at $5 \times 10^{4}$ cells/well in $600 \mu \mathrm{l}$ RPMI-1640 medium supplemented with $1,000 \mathrm{U} / \mathrm{ml}$ penicillin, $1 \mathrm{mg} / \mathrm{ml}$ streptomycin, $2 \mathrm{mM}$ L-glutamine and $10 \%$ fetal bovine serum (Hyclone; GE Healthcare Life Sciences), with or without $5 \mu \mathrm{g} / \mathrm{ml}$ recombinant ULBP1-Fc chimera protein (SinoBiological Inc., Beijing, China), and incubated for $72 \mathrm{~h}$ in a standard $37^{\circ} \mathrm{C}$ environment $\left(5 \% \mathrm{CO}_{2}\right)$. Cell viability was routinely tested by Trypan blue exclusion. Viability, which was calculated as viability $=($ total cells-dead cells $) /$ total cells was $80-90 \%$ after $72 \mathrm{~h}$ of cell culture. Cell-free conditioned medium was removed and stored at $-20^{\circ} \mathrm{C}$ until required for invasion assay and ELISA.

Cell culture. EVT cell line HTR-8/SVneo cells were kindly provided by Dr Charles H. Graham (Queen's University, Kingston, ON, Canada) and cultured in RPMI-1640 medium supplemented with $10 \% \mathrm{FBS}$ in a standard $37^{\circ} \mathrm{C}$ environment $\left(5 \% \mathrm{CO}_{2}\right)$ in an air incubator.

Invasion assay. Cell invasion assays were performed to investigate the effect of ULBP1 on the invasion of HTR-8/SVneo cells. HTR-8/SVneo cells were placed in the upper chamber of an $8-\mu \mathrm{m}$ Transwell plate (Costar, New York, NY, USA) in $80 \mu 1$ growth-factor-reduced Matrigel (1:9; BD Biosciences, San Jose, CA, USA). Inserts were pre-coated with $80 \mu 1$ Matrigel matrix (1:9; BD Biosciences). The different uNK cell conditioned media (cultured with or without the ULBP1 protein, $33 \% \mathrm{v} / \mathrm{v}$ ) was added to the lower chambers. To investigate whether trophoblast invasion was mediated by cytokines secreted by uNK cells, two representative neutralizing antibodies for cytokines that inhibit or stimulate trophoblast cell invasion were added to the 72-h cell supernatants cultured with ULBP1. These antibodies included specific anti-interferon (IFN)- $\gamma$ (mouse anti-human IFN- $\gamma, 10 \mu \mathrm{g} / \mathrm{ml}$; D044-3) for stimulating trophoblast cell invasion and a specific anti-interleukin (IL)-8 neutralizing antibody (goat anti-human IL-8, $0.5 \mu \mathrm{g} / \mathrm{ml}$; MAB208; both R\&D Systems Europe, Ltd.) for inhibition of trophoblast cell invasion. A total of $1 \times 10^{5}$ HTR-8/SVneo cells in $200 \mu \mathrm{l}$ of serum-free medium were plated in the upper chamber, and a total of $600 \mu 1$ media was added in the lower chamber. Following $24 \mathrm{~h}$, the membranes of the Transwell chamber were fixed in methanol and stained with hematoxylin and eosin (OriGene Technologies, Beijing, China). Finally, the number of cells was counted by capturing images of the membrane with an Olympus CKX41 microscope (Olympus Corp.). Each experiment was performed in duplicate in 10 cases. The invasion index was the average number of invaded cells in test filters divided by the average number of invaded cells in control filters for any given experiment.

Flow cytometry. Following 72 h of incubation of uNK cells with or without recombinant ULBP1-Fc $(5 \mu \mathrm{g} / \mathrm{ml})$, surface antigen fluorescence-activated cell sorting analysis was performed to detect the modulation of NKG2D by recombinant ULBP1-Fc. Cells were washed twice with ice-cold PBS. The washes were followed by incubation of the cells with mouse anti-NKG2D antibody (320805; BioLegend, Inc., San Diego, CA, USA) for $30 \mathrm{~min}$ on ice. Subsequent to two washes with PBS, a FACScan flow cytometer (BD Pharmingen, San Diego, CA, USA) was used for analysis. Background staining 


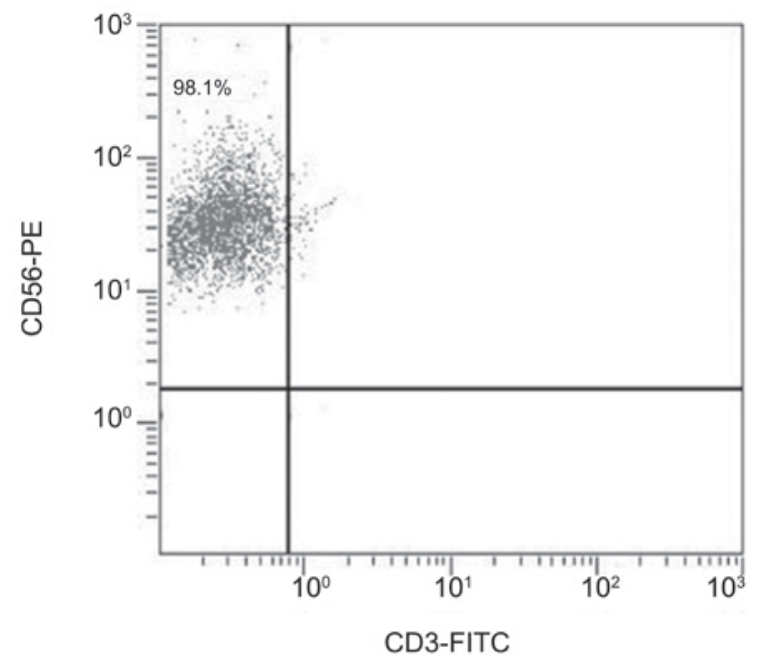

Figure 1. Flow cytometric analysis of the purity of sorted uterine natural killer cells following immunomagnetic selection. The CD56 ${ }^{+} \mathrm{CD} 3-$ cell-enriched isolates were demonstrated to be consistently $>95 \%$ pure.

was estimated after incubation with phycoerythrin-labeled isotype antibodies (400113, 400107 and 400117; $5 \mu$ l per $10^{6}$ cells in $100 \mu 1$ staining volume, all BioLegend, Inc., San Diego, CA, USA). ELISA kits (R\&D Systems Europe, Ltd.) were used to measure tumor necrosis factor (TNF)- $\alpha$ (DTA00C), IFN- $\gamma$ (DIF50), transforming growth factor (TGF)- $\beta 1$ (DB100B), IL-6 (D6050) and IL-8 (D8000C). Cell-free supernatants were analyzed using ELISA following $72 \mathrm{~h}$ incubation of uNK cells with or without recombinant
ULBP1-Fc $(5 \mu \mathrm{g} / \mathrm{ml})$. Each experiment was performed in triplicate in 10 cases.

Statistical analysis. Statistical analyses were performed using SPSS version 17.0 (SPSS, Inc., Chicago, IL, USA). Results are presents as the mean + standard error. Differences between two groups were analyzed using Student's t-test. $\mathrm{P}<0.05$ was considered to indicate a statistically significant result.

\section{Results}

uNK cell isolation. $\mathrm{CD}^{2} 6^{+} \mathrm{CD} 3-$ cell-enriched isolates were demonstrated to be consistently $>95 \%$ pure by flow cytometry (Fig. 1).

$m R N A$ and protein expression levels of ULBPI are upregulated in preeclamptic placentas. RT-qPCR was used to investigate the mRNA expression levels of ULBP1 in preeclamptic and normal placentas. As shown in Fig. 2A, the mRNA expression levels of ULBP1 in preeclampstic placentas were significantly upregulated compared with that in normal placentas $(\mathrm{P}<0.05)$. In agreement with the results of RT-qPCR, western blotting demonstrated that ULBP1 protein expression levels were significantly higher in preeclamptic placentas compared with that of normal placentas $(\mathrm{P}<0.05$; Fig. 2B). Immunocytochemistry demonstrated that ULBP1 was predominantly located in the cytoplasm of STB and that normal and preeclamptic placentas expressed ULBP1; however, ULBP1 expression levels were greater in preeclamptic placentas, as compared with normal placentas (Fig. 2C).
A

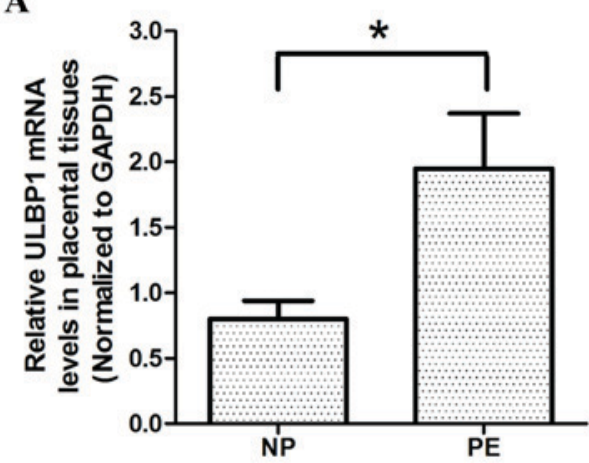

B

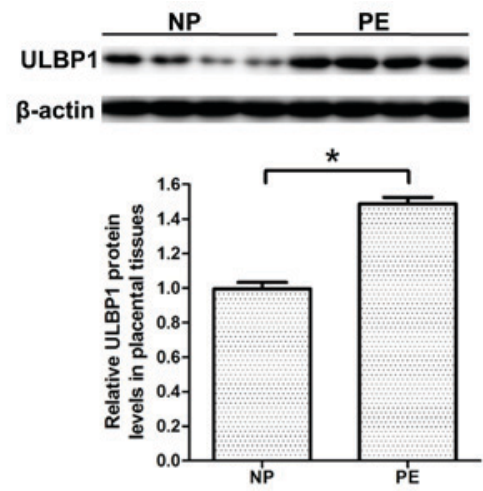

C
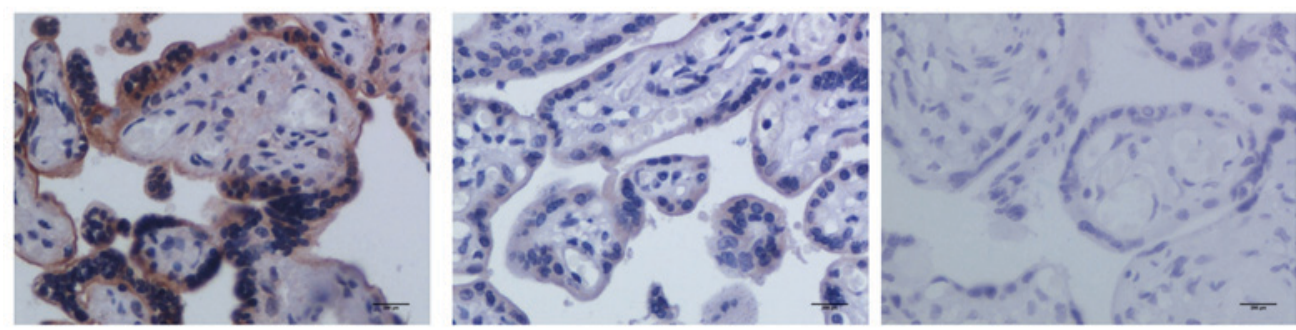

Figure 2. Expression levels of ULBP1 in placentas from pregnant women with PE and women with NP were determined by RT-qPCR, western blotting, and immunohistochemistry analysis. (A) RT-qPCR analysis of ULBP1 mRNA expression levels in placentas from PE and NP women (n=30 for each group). (B) Western blot analysis of ULBP1 protein expression in placentas from PE and NP women. Upper panel, a typical result of western blotting; lower panel, bar chart according to the statistical analysis based on the result of three independently repeated experiments ( $\mathrm{n}=30$ for each group). (C) Immunostaining of ULBP1 in placentas from PE and NP women. Data are presented as the mean + standard error of the mean. Left panel, PE; middle panel, NP; right panel, negative control; scale bars, $200 \mu \mathrm{m}$. ${ }^{*} \mathrm{P}<0.05$, PE vs. NP. ULBP1, unique long 16 binding protein 1; PE, preeclampsia; NP, normal pregnancy; RT-qPCR, reverse transcription-quantitative polymerase chain reaction. 
A

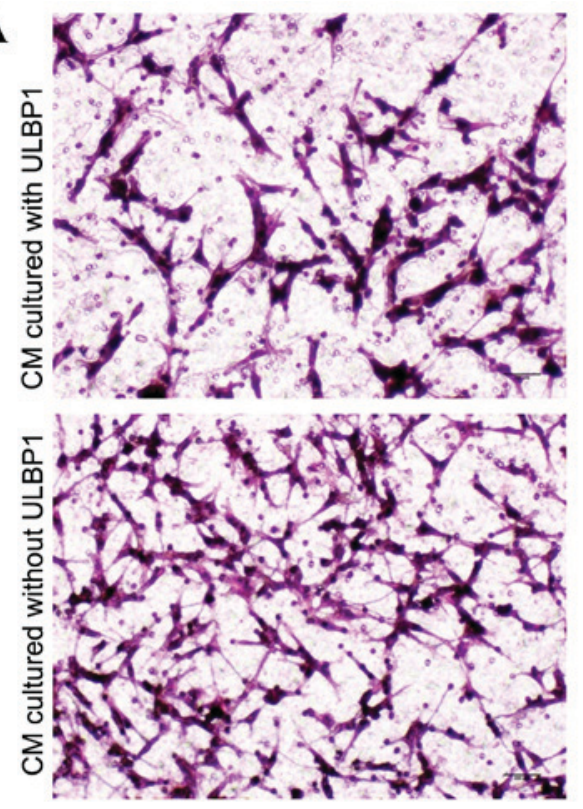

C

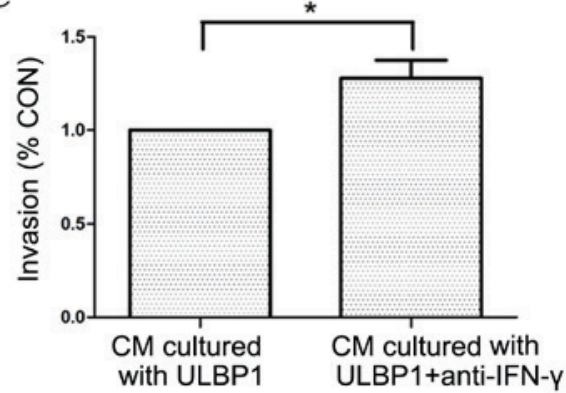

B

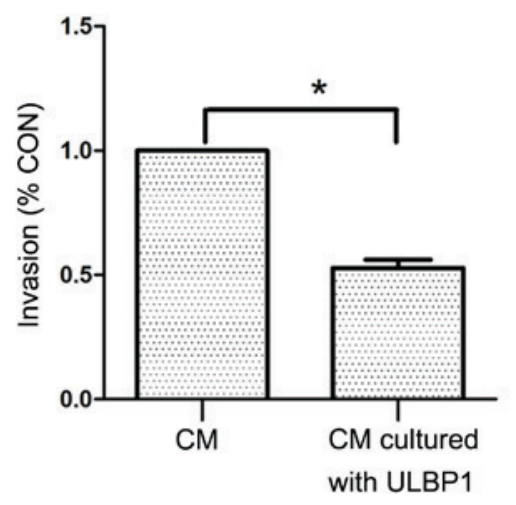

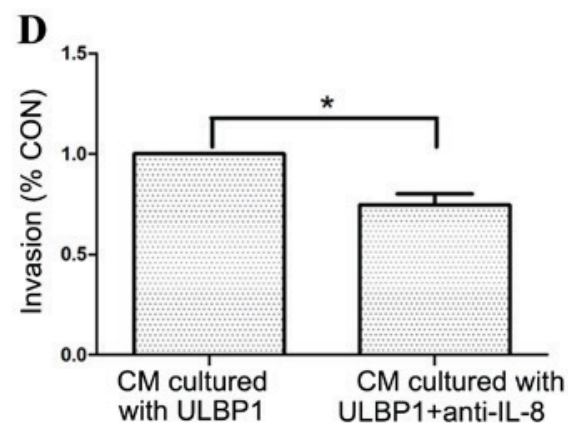

Figure 3. uNK cell supernatants cultured with ULBP1 inhibited HTR-8/SVneo cell invasion. (A) Representative images of invaded cells cultured with different CM in the Transwell invasion assay. Upper panel, the CM is uNK cell supernatants cultured with ULBP1; lower panel, the CM is uNK cell supernatants cultured without ULBP1; scale bars, $200 \mu \mathrm{m}$. Cells were stained with hematoxylin and eosin (magnification, x100). (B) Statistical bar graphs exhibiting the effect of uNK cell supernatants cultured with or without ULBP1 on the invasion of HTR-8/SVneo cells in a Transwell invasion assay. Data are expressed as invasion index ( $\mathrm{n}=10$ in duplicate). (C) Statistical bar graphs exhibiting the effect of the addition of IFN- $\gamma$ neutralizing Ab to the uNK cell supernatants cultured with ULBP1 on invasion of HTR-8/SVneo cells in a Transwell invasion assay ( $\mathrm{n}=10$ in duplicate). (D) Statistical bar graphs exhibiting the effect of the addition of IL-8 neutralizing Ab to the uNK cell supernatants cultured with ULBP1 on invasion of HTR-8/SVneo cells in a Transwell invasion assay (n=10 in duplicate). Data are presented as the mean + standard error of the mean. ${ }^{*} \mathrm{P}<0.05$. uNK, uterine natural killer; ULBP1, unique long 16 binding protein 1 ; HTR-8/SVneo, extravillous trophoblast cell line; IFN, interferon; Ab, antibody; IL, interleukin; CM, condition medium.

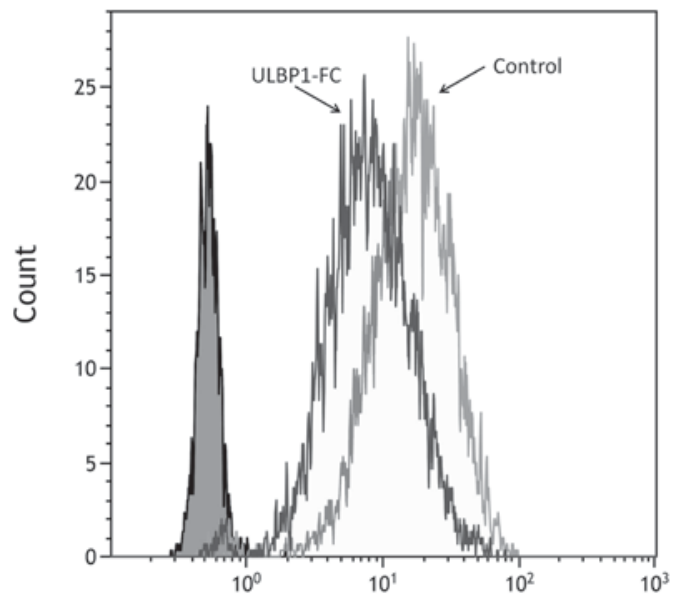

Figure 4. Analysis of natural killer group 2D receptor expression by uNK cells by flow cytometry following incubation of uNK cells with ULBP1-Fc proteins $(5 \mu \mathrm{g} / \mathrm{ml})$ or without ULBP1-Fc proteins (control) for $72 \mathrm{~h}$. The gray filled histogram represents background staining of a corresponding isotype-matched control. uNK, uterine natural killer; ULBP1-Fc, recombinant unique long 16 binding protein $1 \mathrm{Fc}$ chimera.
UNK cell culture supernatants with ULBPI inhibit the invasion of HTR-8/SVneo cells. Following incubation of uNK cells with or without ULBP1 for $72 \mathrm{~h}$, it was possible to determine the effect of ULBP1 on the invasion of EVT. When EVT was cultured in the presence of uNK cell culture supernatants with ULBP1, there was a significant decrease in EVT invasion compared to the cell culture supernatants without ULBP1 ( $\mathrm{P}<0.05$; Fig. 3A and B). Following the addition of specific anti-IFN- $\gamma$ or anti-IL-8 neutralizing antibody to uNK cell culture supernatants with ULBP1, anti-IFN- $\gamma$ neutralizing antibody significantly decreased the inhibitory effect $(\mathrm{P}<0.05$; Fig. $3 \mathrm{C})$ and anti-IL-8 neutralizing antibody enhanced the inhibitory effect of ULBP1 ( $\mathrm{P}<0.05$; Fig. 3D).

ULBP1 stimulates the production of TNF- $\alpha, I F N-\gamma, T G F-\beta 1$, IL6 and IL-8 by downregulating NKG2D expression. ULBP1 decreased surface NKG2D expression levels, as monitored by staining with anti-NKG2D antibody, after incubation of uNK cells for 72 h (Fig. 4). Cell-free supernatants were analyzed, 

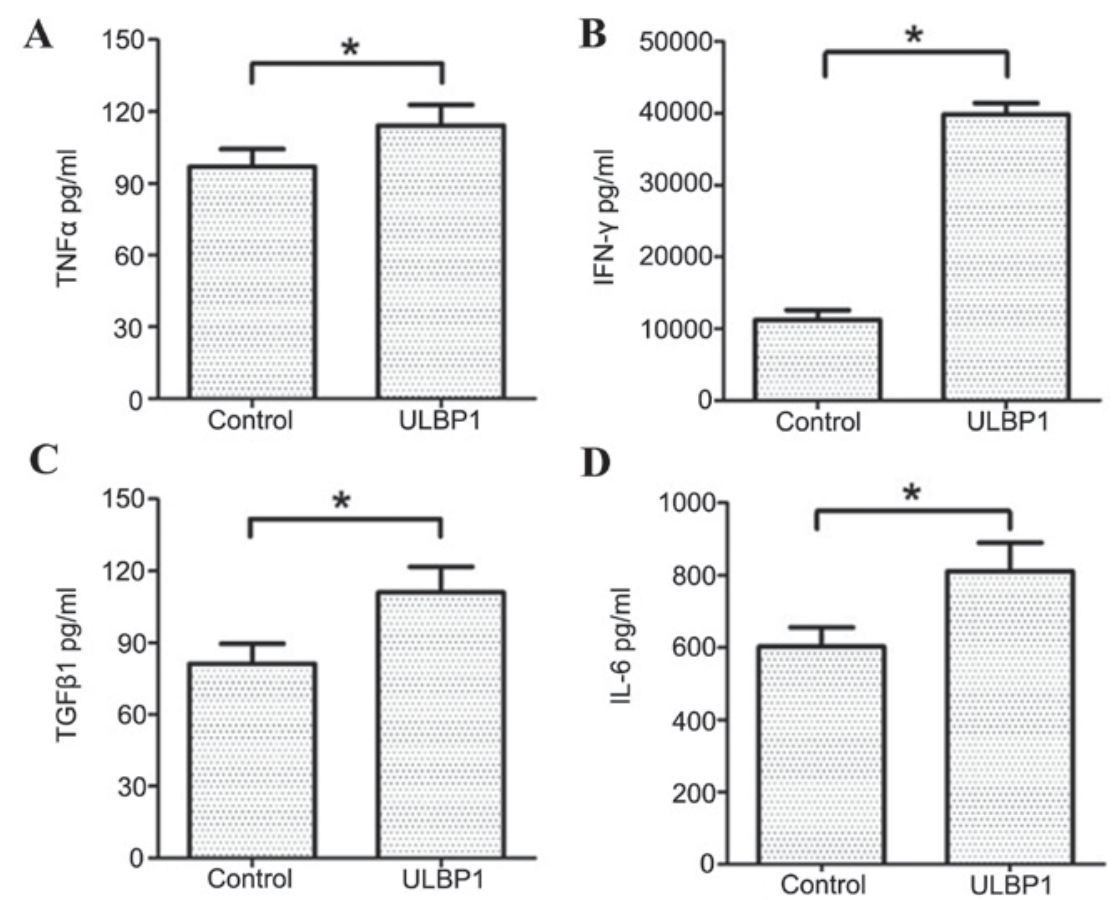

D
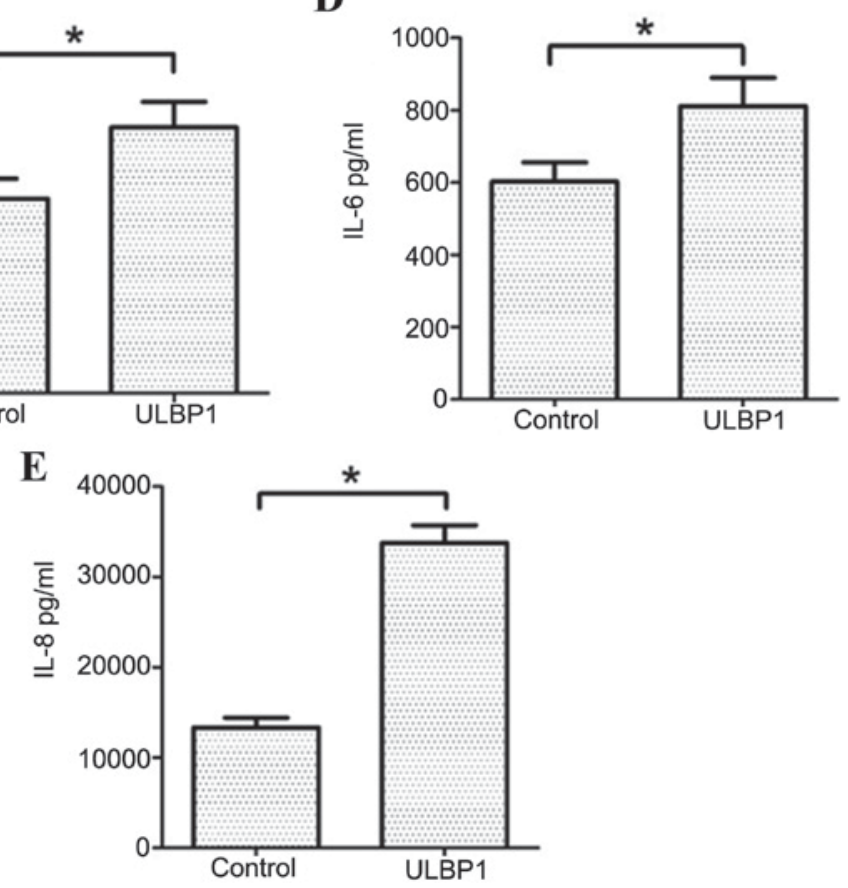

Figure 5. Following incubation of uterine natural killer cells with or without ULBP1-Fc proteins $(5 \mu \mathrm{g} / \mathrm{ml})$ for $72 \mathrm{~h}$, supernatants were analyzed for the presence of (A) TNF- $\alpha$, (B) IFN- $\gamma$, (C) TGF- $\beta 1$, (D) IL- 6 and (E) IL- 8 by ELISA ( $\mathrm{n}=10$ in triplicate). Data are presented as mean + standard error of the mean. ${ }^{*} \mathrm{P}<0.05$ between the two groups. ULBP1-Fc, recombinant unique long 16 binding protein $1 \mathrm{Fc}$ chimera; TNF, tumor necrosis factor; IFN, interferon; TGF, transforming growth factor; IL, interleukin.

using ELISA, following $72 \mathrm{~h}$ incubation for the presence of TNF- $\alpha$, IFN- $\gamma$, TGF- $\beta 1$, IL-6 and IL- 8 . TNF- $\alpha$, IFN- $\gamma$, TGF- $\beta 1$, IL-6 and IL- 8 were all significantly increased in cell-free supernatants incubated with ULBP1 ( $\mathrm{P}<0.05$; Fig. 5) compared with the controls.

\section{Discussion}

uNK cells, CD56 ${ }^{+} \mathrm{CD} 16^{-}$, are distinct from peripheral blood (pb)NK cells and constitute up to $70 \%$ of the decidual leukocyte population in the first half of pregnancy (24). UNK cells are considered to have a cytokine-secreting role rather than a cytotoxic defensive role, which is the predominant role of pbNK cells (25). As previously demonstrated, certain cytokines have the potential to inhibit trophoblast invasion in in vitro invasion assays. These cytokines include TNF- $\alpha$ (26), TGF- $\beta 1$ (9) and IFN- $\gamma$ (27). Certain cytokines stimulate EVT invasion, incuding IL-8 $(8,28)$ and IL-6 (29). A study by Hanna et al (8) demonstrated that UNK cells induced EVT invasion; however, pbNK cells were unable to do this. It is evident that $\mathrm{uNK}$ cells are important for the achievement and maintenance of pregnancy. Although uNK cells have reduced cytotoxic-defensive ability compared with pbNK cells, they do retain low cytotoxic activity $(30,31)$. uNK cells express cytotoxic proteins, including perforin, granzymes $\mathrm{A}$ and $\mathrm{B}$ and granulysin; therefore, uNK cells have cytolytic capacity. This cytotoxic machinery does not result in apoptosis of the invading trophoblast, although apoptosis may occur when responding to infection (32); however, the ability of uNK cells to destroy EVT remains unclear, with some evidence demonstrating that expression of human leukocyte antigen-G by EVT protects EVT from uNK cell attack (33).

The specific gestational period span of uNK cells selected for the present study was based on the research of Lash et al (34), which demonstrated that uNK cell supernatants were able to stimulate EVT invasion at 12-14 weeks gestation; however, they were unable to stimulate EVT invasion at 8-10 weeks gestation. It was suggested that, prior to 10 weeks gestational age, uNK cells had a role in the initial stages of trophoblast-independent SA remodeling and that, after 12 weeks gestational age, uNK cells had a role in regulating EVT invasion. The focus of the present study was on EVT invasion regulated by ULBP1 via uNK cells, therefore, uNK cells at 12-14 weeks were used. 
NK cell function is regulated by a delicate balance of signals initiated from various activating and inhibitory receptors. NKG2D was originally identified, in 1991, as a key activating receptor on all NK cells (35), including uNK cells (8). In humans, NKG2D was first identified as a receptor for major histocompatibility complex class I chain-related A and B and, subsequently, for ULBP 1-5 molecules (36). Binding of ULBPs to NK cells has several biological consequences, including the downregulation of NKG2D expression (37) and the increased production of cytokines and chemokines (38). Flow cytometry analysis demonstrated that ULBP1 downregulated the expression of NKG2D on uNK cells in the present study and, subsequently, all measured cytokines and chemokines secreted by $\mathrm{uNK}$ cells increased. The effect of ULBP1 on uNK cells was the same as pbNK cells (37).

In the present study, ULBP1 expression in the placenta was predominately located in STB, which is consistent with a previous study (18). Additionally, it was demonstrated that ULBP1 expression was upregulated in preeclamptic placenta. A study by Hedlund et al (18) demonstrated that ULBP1-5 were all expressed in the placenta; however, a different study demonstrated differential expression of ULBP1 only. This may have been due to the different binding capacity to NKG2D, which may have induced varying secretory ability (37). Further investigation is required to investigate whether other ULBPs are involved in preeclampsia.

As a ligand of NKG2D, ULBP1 is associated with tumor cells $(39,40)$. The present study demonstrated that ULBP1 is associated with preeclampsia. NKG2D ligands may be upregulated in response to the stresses of pregnancy, such as hypoxia (41). Persistent hypoxia may result in the failure of trophoblasts to differentiate from the proliferative to invasive phenotype, resulting in shallow trophoblast invasion and inadequate transformation of the spiral arteries (42). Considerable evidence supports a role for hypoxia in creating an environment that predisposes women to implantation disorders and preeclampsia $(3,42)$. This may explain the upregulation of ULBP1 in preeclampsia.

ULBP1 is produced and retained in endosomal multivesicular bodies of the STB on exosomes in the first trimester (18). ULBP1 may bind to $\mathrm{UNK}$ cells on decidua via exosomes that are able to carry proteins to target cells. Therefore, there may be an association between ULBP1 and EVT invasion mediated by uNK cells. The present study focused on the secretion from uNK cells following binding of ULBP1. The secretion of cytokines related to the invasion of EVT was detected. The human EVT cell line HTR-8/SVneo, which is widely used to study trophoblast biology $(43,44)$, was utilized in the present study. The expression of NKG2D was downregulated when uNK cells were incubated with ULBP1. Cytokine secretion of TNF- $\alpha$, TGF- $\beta 1$, IFN- $\gamma$, IL- 8 and IL 6 by uNK cells significantly increased in the present study following ULBP1 binding. Human uNK cells produce a variety of cytokines and growth factors, including TNF- $\alpha$, IL-10, granulocyte-macrophage colony-stimulating factor, IL-1, TGF- $\beta 1$, colony-stimulating factor-1, leukemia inhibitory factor, IFN- $\gamma$ (45-47), IL-6 (9), IL-8 and interferon-inducible protein-10 (8), and the roles that these cytokines and growth factors have in EVT invasion has been investigated in previous studies. A study by Bauer et al (26) demonstrated that TNF- $\alpha$ inhibits trophoblast invasion through the elevation of plasminogen activator inhibitor-1. A study by Lash et al (27) demonstrated that IFN- $\gamma$ inhibits EVT cell invasion by a mechanism that involves alterations to apoptosis and protease levels. It was also demonstrated that invasion of EVT cells was inhibited in the presence of TGF- $\beta 1,2$ and 3 , which was associated with a decrease in secreted matrix metalloproteinase (MMP)-9 and urokinase plasminogen activator levels (7). A study by De Oliveira et al (28) concluded that IL-8 stimulates trophoblast invasion by a mechanism that may involve increased secretion of MMP-2 by EVT. IL-6 stimulates trophoblast cell migration and invasion, which may be partly attributable to stimulation of expression of the integrin subunits (29); however, different results on the effect of IL-6 on EVT invasion have been demonstrated, with the study by Champion et al (48) demonstrating that IL-6 has no effect on EVT invasion. Such discrepancies between results may be attributable to differences in cell type and quantification methods used. The present study measured IL-6 as the cell line used was the same as in the study conducted by Jovanović and Vićovac (29). In addition, the present study aimed to indicate the dysfunction of uNK cells in preeclampsia by means of measuring cytokine levels. The levels of cytokines secreted by uNK cells, regardless of their effect on EVT invasion, became unbalanced after ULBP1 binding to uNK.

In conclusion, trophoblast invasion is regulated by crosstalk between trophoblasts and decidual cells in a paracrine and autocrine manner, which involves growth factors, cytokines, chemokines and adhesion molecules. uNK cells produce a wide range of cytokines, chemokines and growth factors that may have diverse effects on trophoblast invasion. Upregulation of ULBP1 disturbs the reproductive balance on the maternal-fetal surface. This balance is complicated and is based on various factors, rather than on one particular factor. The results of the present study demonstrate that ULBP1 is significantly higher in preeclampsia placentas compared with normal placentas. As an active ligand of NK cells, ULBP1 results in the secretion of cytokines that increase EVT invasion. As a result of imbalance, invasion of EVT was inhibited, which is a characteristic of preeclampsia. This finding suggests that inappropriate crosstalk at the fetal-maternal interface disturbs the physiological balance and induces adverse pregnancy. Further investigation is required to determine whether it is possible for ULBP1 to be used as a predictor for other pregnancy complications.

\section{References}

1. Altinbas S, Toğrul C, Orhan A, Yücel M and Danisman N: Increased MPV is not a significant predictor for preeclampsia during pregnancy. J Clin Lab Ana 26: 403-406, 2012.

2. Kaleli I, Kaleli B, Demir M, Yildirim B, Cevahir N and Demir S: Serum levels of neopterin and interleukin-2 receptor in women with severe preeclampsia. J Clin Lab Anal 19: 36-39, 2005.

3. Amash A, Huleihel M, Sheiner E, Sapir O and Holcberg G: Preeclampsia as a maternal vascular disease. Harefush 146: 707-712, 733, 2007.

4. Kharfi A, Giguère Y, Sapin V, Massé J, Dastugue B and Forest JC: Trophoblastic remodeling in normal and preeclamptic pregnancies: Implication of cytokines. Clin Biochem 36: 323-331, 2003.

5. Lala PK and Chakraborty C: Factors regulating trophoblast migration and invasiveness: Possible derangements contributing to pre-eclampsia and fetal injury. Placenta 24: 575-587, 2003 . 
6. Ji L, Brkić J, Liu M, Fu G, Peng C and Wang YL: Placental trophoblast cell differentiation: Physiological regulation and pathological relevance to preeclampsia. Mol Aspects Med 34: 981-1023, 2013.

7. Linzke N, Schumacher A, Woidacki K, Croy BA and Zenclussen AC: Carbon monoxide promotes proliferation of uterine natural killer cells and remodeling of spiral arteries in pregnant hypertensive heme oxygenase- 1 mutant mice. Hypertension 63: 580-588, 2014.

8. Hanna J, Goldman-Wohl D, Hamani Y, Avraham I, Greenfield C, Natanson-Yaron S, Prus D, Cohen-Daniel L, Arnon TI, Manaster I, et al: Decidual NK cells regulate key developmental processes at human fetal-maternal interface. Nat Med 12: 1065-1074, 2006.

9. Lash GE, Otun HA, Innes BA, Bulmer JN, Searle RF and Robson SC: Inhibition of trophoblast cell invasion by TGF $\beta 1$, 2 , and 3 is associated with a decrease in active proteases. Biol Reprod 73: 374-381, 2005.

10. Williams PJ, Bulmer JN, Searle RF, Innes BA and Robson SC: Altered decidual leucocyte populations in the placental bed in pre-eclampsia and foetal growth restriction: A comparison with late normal pregnancy. Reproduction 138: 177-184, 2009.

11. Stallmach T, Hebisch G, Orban P and Lu X: Aberrant positioning of trophoblast and lymphocytes in the feto-maternal interface with pre-eclampsia. Virchows Arch 434: 207-211, 1999.

12. Cao W, Xi X, Hao Z, Li W, Kong Y, Cui L, Ma C, Ba D and He W: RAET1E2, a soluble isoform of the UL16-binding protein RAET1E produced by tumor cells, inhibits NKG2D-mediated NK cytotoxicity. J Biol Chem 282: 18922-18928, 2007.

13. Salih HR, Antropius H, Gieseke F, Lutz SZ, Kanz L, Rammensee HG and Steinle A: Functional expression and release of ligands for the activating immunoreceptor NKG2D in leukemia. Blood 102: 1389-1396, 2003.

14. Pende D, Rivera P, Marcenaro S, Chang CC, Biassoni R, Conte R, Kubin M, Cosman D, Ferrone S, Moretta L and Moretta A: Major histocompatibility complex class I-related chain A and UL16-binding protein expression on tumor cell lines of different histotypes: Analysis of tumor susceptibility to NKG2D-dependent natural killer cell cytotoxicity. Cancer Res 62: 6178-6186, 2002.

15. Mincheva-NilssonL,Nagaeva O,ChenT,StendahlU,Antsiferova J, Mogren I, Hernestål J and Baranov V: Placenta-derived soluble MHC class I chain-related molecules down-regulate NKG2D receptor on peripheral blood mononuclear cells during human pregnancy: A possible novel immune escape mechanism for fetal survival. J Immunol 176: 3585-3592, 2006.

16. Upshaw JL and Leibson PJ: NKG2D-mediated activation of cytotoxic lymphocytes: Unique signalling pathways and distinct functional outcomes. Semin Immunol 18: 167-175, 2006.

17. Borchers MT, Harris NL, Wesselkamper SC, Vitucci M and Cosman D: NKG2D ligands are expressed on stressed human airway epithelial cells. Am J Physiol 291: L222-L231, 2006.

18. Hedlund M, Stenqvist AC, Nagaeva O, Kjellberg L, Wulff M, Baranov V and Mincheva-Nilsson L: Human placenta expresses and secretes NKG2D ligands via exosomes that down-modulate the cognate receptor expression: Evidence for immunosuppressive function. J Immunol 183: 340-351, 2009.

19. Meng $T$, Chen $H$, Sun $M$, Wang $H$, Zhao $G$ and Wang $X$ : Identification of differential gene expression profiles in placentas from preeclamptic pregnancies versus normal pregnancies by DNA microarrays. OMICS 16: 301-311, 2012.

20. Steegers EA, von Dadelszen P, Duvekot JJ and Pijnenborg R: Pre-eclampsia. Lancet 376: 631-644, 2010.

21. Livak KJ and Schmittgen TD: Analysis of relative gene expression data using real-time quantitative PCR and the 2(-Delta Delta C(T)) method. Methods 25: 402-408, 2001

22. Lash GE, Schiessl B, Kirkley M, Innes BA, Cooper A, Searle RF, Robson SC and Bulmer JN: Expression of angiogenic growth factors by uterine natural killer cells during early pregnancy. J Leukoc Biol 80: 572-580, 2006.

23. Vassiliadou N and Bulmer JN: Functional studies of human decidua in spontaneous early pregnancy loss: Effect of soluble factors and purified CD56+ lymphocytes on killing of natural killer- and lymphokine-activated killer-sensitive targets. Biol Reprod 58: 982-987, 1998.

24. Moffett-King A: Natural killer cells and pregnancy. Nat Rev Immunol 2: 656-663, 2002
25. Tabiasco J, Rabot M, Aguerre-Girr M, El Costa H, Berrebi A, Parant O, Laskarin G, Juretic K, Bensussan A, Rukavina D and Le Bouteiller P: Human decidual NK cells: Unique phenotype and functional properties-a review. Placenta 27 (Suppl A): S34-S39, 2006.

26. Bauer S, Pollheimer J, Hartmann J, Husslein P, Aplin JD and Knöfler M: Tumor necrosis factor-alpha inhibits trophoblast migration through elevation of plasminogen activator inhibitor-1 in first-trimester villous explant cultures. J Clin Endocrinol Metab 89: 812-822, 2004

27. Lash GE, Otun HA, Innes BA, Kirkley M, De Oliveira L, Searle RF, Robson SC and Bulmer JN: Interferon-gamma inhibits extravillous trophoblast cell invasion by a mechanism that involves both changes in apoptosis and protease levels. FASEB J 20: 2512-2518, 2006.

28. De Oliveira LG, Lash GE, Murray-Dunning C, Bulmer JN, Innes BA, Searle RF, Sass N and Robson SC: Role of interleukin 8 in uterine natural killer cell regulation of extravillous trophoblast cell invasion. Placenta 31: 595-601, 2010.

29. Jovanović M and Vićovac L: Interleukin-6 stimulates cell migration, invasion and integrin expression in HTR-8/SVneo cell line. Placenta 30: 320-328, 2009.

30. King A, Birkby C and Loke YW: Early human decidual cells exhibit NK activity against the K562 cell line but not against first trimester trophoblast. Cell Immunol 118: 337-344, 1989.

31. Ritson A and Bulmer JN: Isolation and functional studies of granulated lymphocytes in first trimester human decidua. Clin Exp Immunol 77: 263-268, 1989.

32. Le Bouteiller P, Siewiera J, Casart Y, Aguerre-Girr M, El Costa H, Berrebi A, Tabiasco J and Jabrane-Ferrat N: The human decidual NK-cell response to virus infection: What can we learn from circulating NK lymphocytes? J Reprod Immunol 88: 170-175, 2011.

33. Rouas-Freiss N, Gonçalves RM, Menier C, Dausset J and Carosella ED: Direct evidence to support the role of HLA-G in protecting the fetus from maternal uterine natural killer cytolysis. Proc Natl Acad Sci USA 94: 11520-11525, 1997.

34. Lash GE, Otun HA, Innes BA, Percival K, Searle RF, Robson SC and Bulmer JN: Regulation of extravillous trophoblast invasion by uterine natural killer cells is dependent on gestational age. Hum Reprod 25: 1137-1145, 2010.

35. Houchins JP, Yabe T, McSherry C and Bach FH: DNA sequence analysis of NKG2, a family of related cDNA clones encoding type II integral membrane proteins on human natural killer cells. J Exp Med 173: 1017-1020, 1991.

36. González S, López-Soto A, Suarez-Alvarez B, López-Vázquez A and López-Larrea C: NKG2D ligands: Key targets of the immune response. Trends Immunol 29: 397-403, 2008.

37. Song H, Kim J, Cosman D and Choi I: Soluble ULBP suppresses natural killer cell activity via down-regulating NKG2D expression. Cell Immunol 239: 22-30, 2006.

38. Kubin M, Cassiano L, Chalupny J, Chin W, Cosman D, Fanslow W, Müllberg J, Rousseau AM, Ulrich D and Armitage R: ULBP1, 2, 3: Novel MHC class I-related molecules that bind to human cytomegalovirus glycoprotein UL16, activate NK cells. Eur J Immunol 31: 1428-1437, 2001.

39. Kim SJ, Ha GH, Bae JH, Kim GR, Son CH, Park YS, Yang K, Oh SO, Kim SH and Kang CD: COX-2- and endoplasmic reticulum stress-independent induction of ULBP-1 and enhancement of sensitivity to NK cell-mediated cytotoxicity by celecoxib in colon cancer cells. Exp Cell Res 330: 451-459, 2015.

40. Cho H, Chung JY, Kim S, Braunschweig T, Kang TH, Kim J, Chung EJ, Hewitt SM and Kim JH: MICA/B and ULBP1 NKG2D ligands are independent predictors of good prognosis in cervical cancer. BMC Cancer 14: 957, 2014.

41. Weier JF, Weier HU, Jung CJ, Gormley M, Zhou Y, Chu LW, Genbacev O, Wright AA and Fisher SJ: Human cytotrophoblasts acquire aneuploidiesas they differentiate to an invasive phenotype. Dev Biol 279: 420-432, 2005.

42. Caniggia I, Mostachfi H, Winter J, Gassmann M, Lye SJ, Kuliszewski $M$ and Post M: Hypoxia-inducible factor-1 mediates the biological effects of oxygen on human trophoblast differentiation through TGFbeta(3). J Clin Invest 105: 577-587, 2000.

43. Suman P, Godbole G, Thakur R, Morales-Prieto DM, Modi DN, Markert UR and Gupta SK: AP-1 transcription factors, mucin-type molecules and MMPs regulate the IL-11 mediated invasiveness of JEG-3 and HTR-8/SVneo trophoblastic cells. PLoS One 7: e29745, 2012. 
44. Graham CH,Hawley TS, Hawley RG, MacDougall JR, Kerbel RS, Khoo N and Lala PK: Establishment and characterization of first trimester human trophoblast cells with extended lifespan. Exp Cell Res 206: 204-211, 1993.

45. Jokhi PP, King A, Sharkey AM, Smith SK and Loke YW: Screening for cytokine messenger ribonucleic acids in purified human decidual lymphocyte populations by the reverse-transcriptase polymerase chain reaction. J Immunol 153: 4427-4435, 1994.

46. Jokhi PP, King A and Loke YW: Cytokine production and cytokine receptor expression by cells of the human first trimester placental-uterine interface. Cytokine 9: 126-137, 1997.
47. Rieger L, Kammerer U, Hofmann J, Sütterlin M and Dietl J: Choriocarcinoma cells modulate the cytokine production of decidual large granular lymphocytes in coculture. Am J Reprod Immunol 46: 137-143, 2001.

48. Champion H, Innes BA, Robson SC, Lash GE and Bulmer JN: Effects of interleukin- 6 on extravillous trophoblast invasion in early human pregnancy. Mol Hum Reprod 18: 391-400, 2012. 